\title{
FIRST ON-SUN TESTS OF A CENTRIFUGAL PARTICLE RECEIVER SYSTEM
}

\author{
Miriam Ebert \\ German Aerospace Center, \\ Institute of Solar Research \\ 70569 Stuttgart, Germany \\ miriam.ebert@dlr.de \\ Jens Rheinländer \\ German Aerospace Center, \\ Institute of Solar Research \\ 70569 Stuttgart, Germany \\ jens.rheinlaender@dlr.de \\ Marcel Sibum \\ German Aerospace Center, \\ Institute of Solar Research \\ 52428 Juelich, Germany \\ marcel.sibum@dlr.de
}

\author{
Lars Amsbeck \\ German Aerospace Center, \\ Institute of Solar Research \\ 70569 Stuttgart, Germany \\ lars.amsbeck@dlr.de \\ Bärbel Schlögl-Knothe \\ German Aerospace Center, \\ Institute of Solar Research \\ 52428 Juelich, Germany \\ baerbel.schloegl@dlr.de \\ Hannes Stadler \\ German Aerospace Center, \\ Institute of Solar Research \\ 52428 Juelich, Germany \\ hannes.stadler@dlr.de
}

\author{
Reiner Buck \\ German Aerospace Center, \\ Institute of Solar Research \\ 70569 Stuttgart, Germany \\ reiner.buck@dlr.de \\ Stefan Schmitz \\ German Aerospace Center, \\ Institute of Solar Research \\ 52428 Juelich, Germany \\ stefan.schmitz@dlr.de \\ Ralf Uhlig \\ German Aerospace Center, \\ Institute of Solar Research \\ 70569 Stuttgart, Germany \\ ralf.uhlig@dlr.de
}

\section{ABSTRACT}

One direct absorption receiver concept currently investigated at the DLR is the Centrifugal Particle Receiver $\left(\right.$ CentRec $\left.{ }^{\circledR}\right)$. Successful tests and promising results of this receiver design have been achieved in a Proof-of-Concept scale with $7.5 \mathrm{~kW}$ thermal power and $900^{\circ} \mathrm{C}$ particle temperature in 2014. Based on these results the prototype has been scaled up to $2.5 \mathrm{MW}$ thermal power for a future pilot plant. Lab tests have been carried out with infrared heaters. In a next step the prototype has been prepared to be tested on-sun in a test setup in the Juelich Solar Tower, Germany. The tests aim to demonstrate high temperature operation and to evaluate the performance of the system.

The test setup consists of a centrifugal receiver integrated into the tower and a closed loop particle transport system. The transport system includes an air cooling system to cool down the particles at the receiver outlet, cold particle storage, belt bucket elevator, hopper and particle metering system. While the $2.5 \mathrm{MW}_{\text {th }}$ receiver prototype has been developed in a former project, the further infrastructure for the on-sun tests needed to be designed, manufactured and installed. The system is equipped with measurement instrumentation, data acquisition system and control software. Manufacturing of all main components has been completed. Installation of the test setup started in November 2016 and finished in June 2017. Cold and hot commissioning have been carried out from July 2017 until September 2017. On-sun tests started in September 2017. Receiver tests up to $775^{\circ} \mathrm{C} / 1,430^{\circ} \mathrm{F}$ receiver outlet temperature and more than $900^{\circ} \mathrm{C} / 1,650^{\circ} \mathrm{F}$ particle temperature in the receiver have already been achieved. Tests up to $900^{\circ} \mathrm{C}$ particle outlet temperature are planned at different load levels and will be conducted until summer 2018 .

This paper describes the test setup for a centrifugal particle receiver system, presenting design, installation and commissioning of the system. It presents test results of first onsun tests and gives an outlook on further steps regarding solar tests planned for 2018 .

\section{INTRODUCTION}

One receiver concept currently investigated at the DLR is the so-called Centrifugal Particle Receiver (CentRec $®)$. This technology uses inexpensive sand-like solid particles both as a heat transfer and as a storage medium. The properties of the used particles are given in TABLE 1.

The incoming solar radiation is directly absorbed by the particles. This allows high incoming flux densities and operating temperatures up to $1,000^{\circ} \mathrm{C} / 1,800^{\circ} \mathrm{F}$ at very high efficiencies. 
Particle inlet and outlet temperatures can be chosen for an optimal configuration depending on the application. Minimum values are normally given by the application. For instance, a steam turbine with a feed water pre-heating temperature of $300^{\circ} \mathrm{C} / 572^{\circ} \mathrm{F}$ results in a particle inlet temperature of the receiver higher than the feed water temperature; a life steam temperature of $550^{\circ} \mathrm{C} / 1,022^{\circ} \mathrm{F}$ requires a particle outlet temperature above that value. Higher values than the minimum values are beneficial to reduce heat exchanger costs through higher driving temperature differences. Larger temperature differences between particle receiver outlet and inlet temperatures additionally enhance the storage capacity of the particles. At the same time higher temperatures in general increase losses and, to a smaller degree, result in higher material costs as better materials have to be chosen.

The CentRec ${ }^{\circledR}$ technology enables a solution for different high temperature applications, e.g. process heat as hot gas or steam [1], steam for conventional power blocks or high temperature $\mathrm{sCO}_{2}$ for future advanced power blocks. It allows a 24-hour operation, along with guaranteed energy availability and process stability through storage and a conventional backup system using small amounts of conventional fuel when no solar radiation is available for longer periods.

Successful tests and promising results of this receiver design have been achieved at Proof-of-Concept scale with $7.5 \mathrm{~kW}_{\text {th }}$ thermal power [2]. Based on these results the prototype has been scaled up to $2.5 \mathrm{MW}_{\text {th }}$ for a future pilot plant [3]. Lab tests of the $2.5 \mathrm{MW}_{\text {th }}$ receiver have been carried out with infrared heaters [4]. In a next step, the prototype has been prepared to be tested on-sun in a test setup in the research platform of the Juelich Solar Tower (STJ) in Juelich, Germany [5]. Tests up to $500 \mathrm{~kW}_{\text {th }}$ power and $900^{\circ} \mathrm{C} / 1,650^{\circ} \mathrm{F}$ receiver outlet temperature are planned.

TABLE 1 PARTICLE PROPERTIES

\begin{tabular}{|l|c|}
\hline Material & Sintered Bauxite \\
\hline Manufacture description & Saint-Gobain Proppants 16/30 \\
\hline Median particle diameter & $0.917 \mathrm{~mm} / 0.038 \mathrm{inch}$ \\
\hline Bulk density & $2.02 \mathrm{~g} / \mathrm{cc} / 125 \mathrm{lbs} / \mathrm{ft}^{3}$ \\
\hline
\end{tabular}

\section{NOMENCLATURE}

$\begin{array}{ll}\mathrm{T}_{\text {REC,IN }} & \text { receiver inlet temperature } \\ \mathrm{T}_{\text {REC,OUT }} & \text { receiver outlet temperature } \\ \mathrm{T}_{\text {REC,SURFACE }} & \text { receiver temperature at lower end of rotating } \\ & \text { cylinder } \\ \mathrm{n}_{\text {Helio }} & \text { Heliostats focused on receiver } \\ \omega_{\text {REC }} & \text { rotational speed of the receiver } \\ \dot{m}_{\text {particles }} & \text { particle mass flow } \\ \varepsilon & \text { emissivity }\end{array}$

\section{SYSTEM DESCRIPTION}

\section{Receiver}

The basic concept of the receiver and a first prototype are described in [2]. A more detailed description and presentation of a scaled up receiver prototype with a power of $2.5 \mathrm{MW}_{\text {th }}$ is given in [3]. Main properties of the prototype are additionally given in TABLE 2. The prototype is to be tested at $500 \mathrm{~kW}_{\text {th }}$ due to constraints of the heliostat field that is designed for a lower flux atmospheric air receiver at about double the height of the test platform of the Juelich solar tower.

The concept of the receiver shown in FIGURE 1 is based on a rotating cylinder with an inclination of the rotation axis towards horizontal. Particles enter into the receiver in the upper part at an inlet on the rotational axis and are accelerated by a feeding cone to the circumferential speed of the receiver. The particles form a particle film on the inner surface of the cylindrical absorber cavity. They move along the inner wall towards the outlet governed by gravity and centrifugal forces with a controllable residence time. Concentrated sunlight enters through the aperture and heats up the particles through direct absorption. The particles leave the rotating receiver part at the lower end where a stationary collector ring catches all particles and conducts them to an outlet piping into the storage [3]. The rotating cylinder is insulated on the inside so that the moving parts like bearing and drive of the receiver on the outer side of the cylinder stay cold during operation.

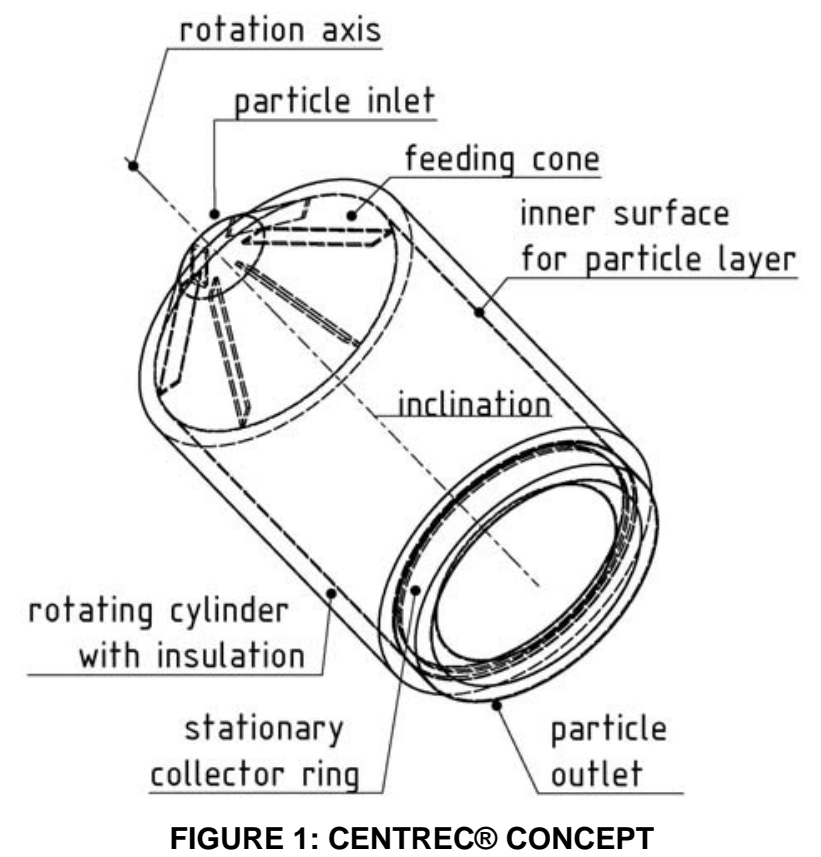

TABLE 2 SPECIFICATIONS OF PROTOTYPE

\begin{tabular}{|c|c|c|}
\hline \multicolumn{2}{|c|}{ Aperture area and diameter } & $1 \mathrm{~m}^{2}, 1.13 \mathrm{~m}$ \\
\hline \multicolumn{2}{|c|}{ Rotation axis inclination } & $45^{\circ}$ \\
\hline \multirow[t]{2}{*}{ Thermal power } & Validation test setup & $500 \mathrm{~kW}_{\text {th }}$ \\
\hline & STJ Commercial setup & $2,500 \mathrm{~kW}_{\text {th }}$ \\
\hline \multirow{2}{*}{$\begin{array}{l}\text { Receiver outlet } \\
\text { temperature }\end{array}$} & Minimum & $900^{\circ} \mathrm{C} / 1,650^{\circ} \mathrm{F}$ \\
\hline & Design & $1,000^{\circ} \mathrm{C} / 1,830^{\circ} \mathrm{F}$ \\
\hline \multicolumn{2}{|c|}{$\begin{array}{l}\text { Particle mass flow at } 2.5 \mathrm{MW}_{\text {th }}, 200^{\circ} \mathrm{C} / \\
900^{\circ} \mathrm{C} \text { receiver inlet/outlet temperature }\end{array}$} & $3 \mathrm{~kg} / \mathrm{s}$ \\
\hline \multicolumn{2}{|c|}{ Rotational speed } & aprox. $45 \mathrm{rpm}$ \\
\hline
\end{tabular}


The receiver outlet temperature can be controlled by changing the rotational speed of the receiver and thus the residence time of the particles. A more detailed description of the particle film behavior and control is given in [4]. One important detail is a wire mesh which is point-welded on the inner surface in order to provide a rough surface for the particle layer (see FIGURE 1). Particles move into the wire mesh and are blocked in the axial direction by the tangential wires of the mesh because the wire diameter is larger than the average particle diameter of $1 \mathrm{~mm}$. By this, a rough surface is formed with the same friction coefficient between the moving particles and the wall as between two particle layers. A photo of the wire is given in FIGURE 9.

The particle film is very sensitive to small changes in the circumferential speed. These changes can be caused by changes in the rotational speed as well as caused by slightly different radiuses due to tolerances of the inner surface for the particle layer from the manufacturing. Additionally, every particle mass flow requires its corresponding rotational speed. For high mass flows particles move over the complete circumference of the receiver, even despite slight differences of the radius due to manufacturing tolerances. Therefore the operation mode for high mass flows uses one constant rotational speed according to the given mass flow.

For very low mass flows the system is more sensitive on changes in the circumferential speed as fewer particles are involved. Inhomogeneities in the particle film could be observed at low mass flows, e.g. caused by tolerances from manufacturing. To guarantee a homogeneous particle movement over the complete circumference of the receiver, a simple and reliable operation mode has been found by periodically changing the rotational speed between two slightly different values: a higher rotational speed where all particles stick to the wall due to the high centrifugal force (the particle film is "frozen") and a lower rotational speed resulting in reduced centrifugal forces where the particle film moves towards the outlet. Depending on the particle mass flow and the incoming radiation the receiver outlet temperature and the thickness of the film can then be adjusted by maintaining a longer or shorter dwell time on the upper rotational speed value. The smaller the mass flow and the lower the incoming radiation the higher the dwell time needs to be set. For a mass flow of $0.07 \mathrm{~kg} / \mathrm{s}$ the following values have been identified as good point to start: $45.4 \mathrm{rpm}$ for the upper value with a dwell time of 25 seconds and $43.6 \mathrm{rpm}$ for the lower value. The course of the rotational speed for an exemplary test day is given in FIGURE 8.

Direct absorption and homogenization due to the rotation of the receiver allow high fluxes and high transients on the receiver as no material temperature in the receiver limits the flux. Thus, fewer restrictions on operation need to be considered and high flux densities of more than $1 \mathrm{MW}_{\mathrm{th}} / \mathrm{m}^{2}$ on the inner wall can be applied. FIGURE 2 shows the CentRec ${ }^{\circledR}$ receiver prototype during installation in DLR's Juelich Solar Tower, Germany.

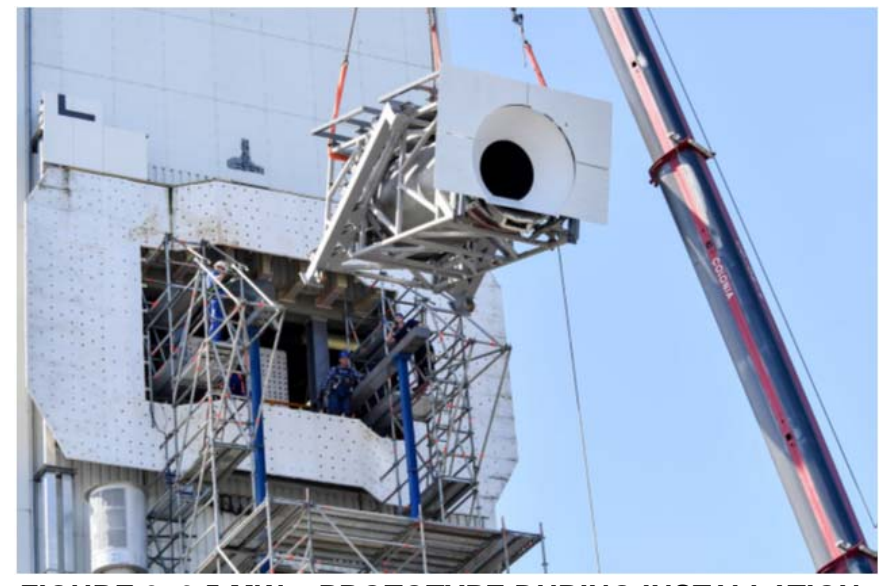

FIGURE 2: $2.5 \mathrm{MW}_{\text {TH }}$ PROTOTYPE DURING INSTALLATION IN THE JUELICH SOLAR TOWER

\section{Particle system}

A test infrastructure has been developed, manufactured and built to provide a closed loop particle system to test the CentRec $\AA$ receiver. A flow sheet and an artistic CAD view of the infrastructure are given in FIGURE 3 and FIGURE 4.

The closed particle loop starts in a cold storage at the bottom of the tower. The storage with a volume of approximately $4 \mathrm{~m}^{3}$ can store the total amount of all particles in the system. A valve at the outlet of the storage on the bottom side controls the mass flow of particles fed to the vertical transport system. The valve is based on the principle of a clamshell gate, but embedded inside a housing. The transport system is a belt bucket elevator with $32 \mathrm{~m} / 105 \mathrm{ft}$ height. From the outlet of the belt bucket elevator the particles move driven by gravity through a piping with the required inclination and end in a hopper just before entering the receiver. The hopper can contain up to $350 \mathrm{~kg}$ of particles. A pinch valve allows controlling the mass flow into the receiver. At the outlet of the receiver the hot particles fall down through an inclined piping into a vertical piping and then back to the cold storage. Meanwhile the particles are cooled down in counter current in the vertical piping with an air blower installed at the bottom of the tower. Hot air leaves the system through an air vent installed at the upper end of the vertical piping. The air vent includes dust removal realized by a metallic mesh with a mesh size of $0.3 \mathrm{~mm}$.

For the prototype system an additional emergency system has been installed. In case of emergency (black out or emergency stop of the system due to problems) the receiver driven by an electric motor stops suddenly. As a consequence, a part of the particles in the receiver can escape through the aperture and thus leave the closed particle loop. In this case particles are collected by an hopper installed just below the aperture and stored in an insulated container on the ground. The receiver and all peripheral installations on the tower are protected with a partly passive insulated, partly actively watercooled front protection shield. 
The receiver and the test system are positioned on top of a scaffold in front of the research platform at $26 \mathrm{~m} / 85 \mathrm{ft}$ height of the Juelich Solar Tower. The scaffold has been needed due to space limitations inside the research platform.

The system has been designed and manufactured starting with pre-studies in 2015. Installation in Juelich started in November 2016 and has been finished in June 2017.

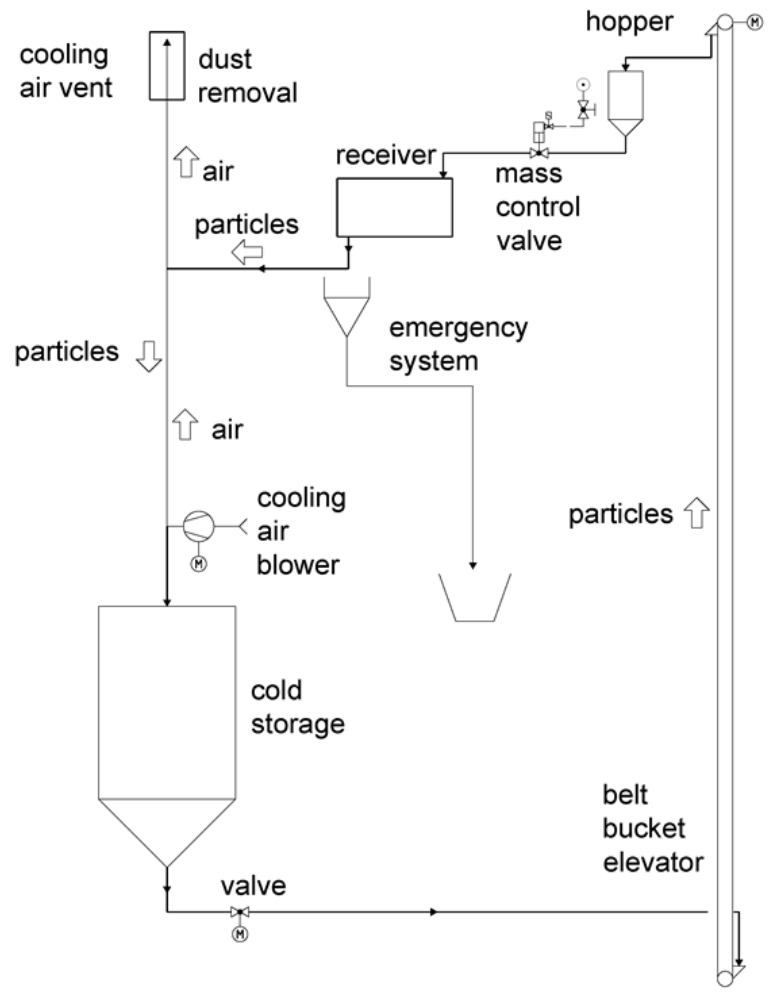

FIGURE 3: FLOW SHEET OF PARTICLE SYSTEM

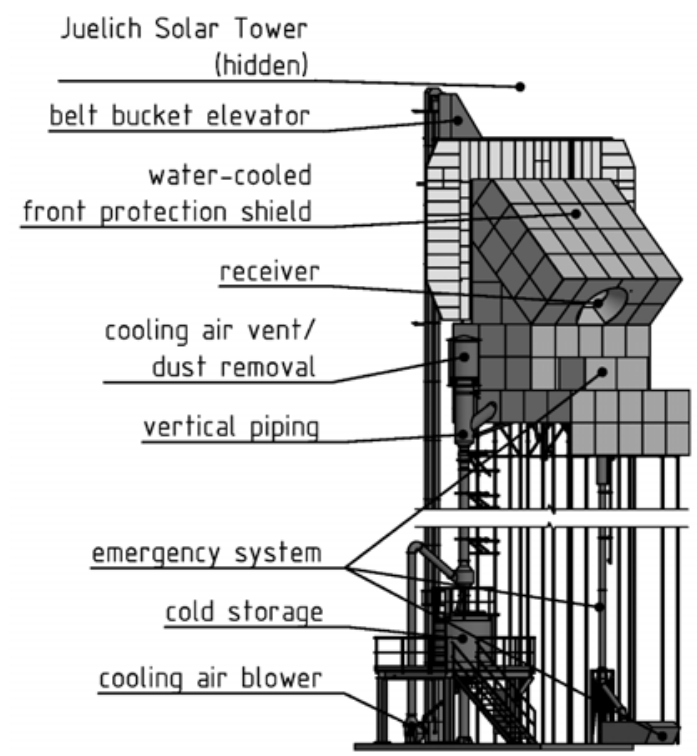

FIGURE 4: CAD DRAWING OF CENTREC $®$ SYSTEM (INTERRUPTED VIEW OF TOWER)

\section{Measurement and control system}

The system is equipped with more than 250 sensors, mainly thermocouples, from which approximately 100 are installed in the rotating part of the receiver to monitor, control and evaluate the temperatures of the system. The positions of the most important thermocouples are shown in Figure 5. The receiver inlet temperature $\mathrm{T}_{\mathrm{REC}, \mathrm{IN}}$ is measured inside the hopper at the inlet of the receiver. The receiver outlet temperature $T_{\text {REC,OUT }}$ is measured inside a piping behind the outlet of the stationary collector ring. Inside the rotating cylinder temperatures are measured on the rear side of a metallic surface on which the particle layer moves down. Thus, these temperatures correspond closely to the particle temperatures inside the receiver. 12 of these thermocouples are equally distributed along the circumference about $100 \mathrm{~mm}$ above the end of the rotating cylinder. These thermocouples measure the temperature $\mathrm{T}_{\mathrm{REC}, \mathrm{SURFACE}}$, corresponding closely to the particle temperature before leaving the cylinder. Only four of the twelve thermocouples worked properly during on-sun tests. All four thermocouples are adjoining so that only one third of the circumference of the inner surface is covered with sensors.

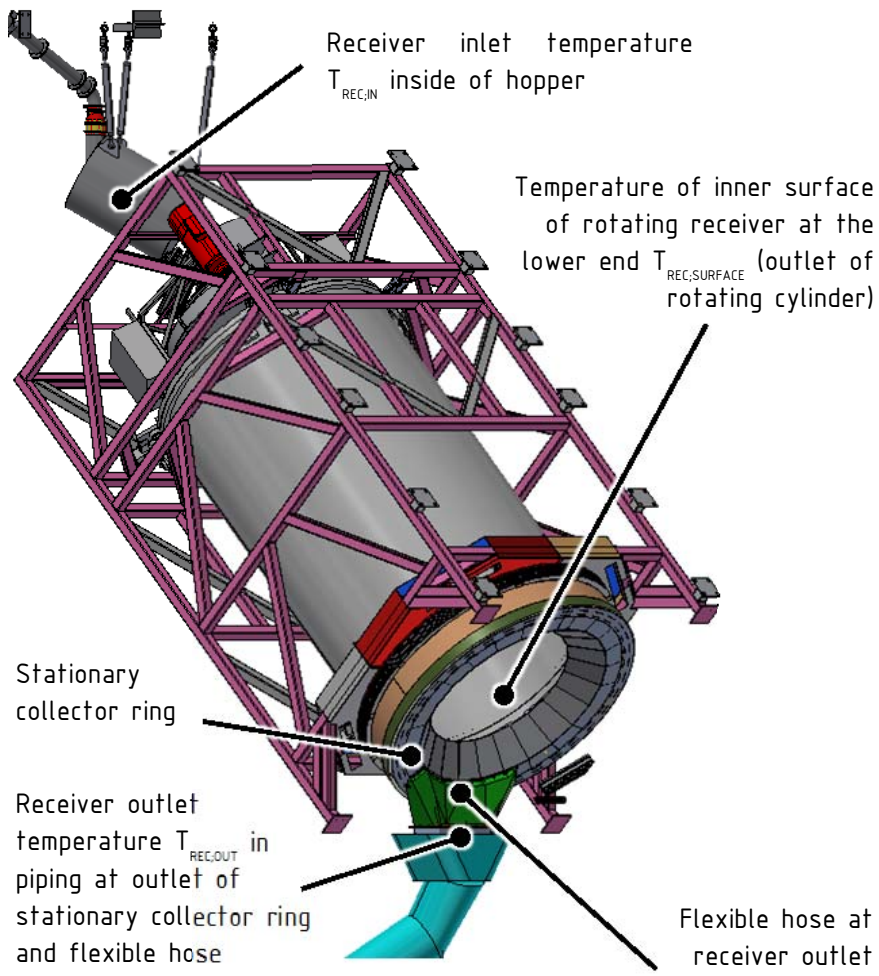

FIGURE 5: POSITION OF MAIN TEMPERATURE SENSORS

The mass flow of the particles $\dot{m}_{\text {particles }}$ is measured at the inlet of the receiver. It is determined by evaluating the weight changes of the hopper over time using force sensors in the support of the hopper. This gravitational measurement is possible while particles are fed into the receiver but no particles are entering the hopper. To allow this procedure the particle transport works in intervals at higher mass flows than the 
receiver mass flow. An automatic control implemented into the system opens and closes the clamshell gate under the cold storage when the hopper exceeds the lower or upper limit to start and stop the refilling with particles. Meanwhile the belt bucket elevator runs continuously to avoid start-stop-operation which decreases the lifetime of the elevator. This operation mode allow a continuously operation of the system without any limitation due to the capacity of the hopper and allows a reliable mass flow measurement.

In the bearings of the receiver the forces are measured to be able to reduce imbalances. Additional sensors are a rotational speed sensor for the receiver, level sensors in the cold storage, position feedback of the valves, pressure sensors and flow control sensors to monitor cooling air and cooling water. All data are collected in a data acquisition system based on a PLC. Visualization and user interface are implemented in LabView [6]. A meteorological station and the heliostat field control are independent systems running in parallel. For the case of emergency shutdowns, the cooling system and the CentRec ${ }^{\circledR}$ system are hard wired to defocus the heliostat field.

Additionally, infrared and optical supervision of the system is carried out with two infrared cameras in the heliostat field and several digital cameras in the heliostat field and inside of the research platform.

The determination of solar flux density at receiver aperture and its spatial resolution is possible by shifting the focus aim point temporarily from the center of the receiver aperture onto the nearby passive insulated protection shield [7], similar to a measurement technique proposed in [8]. The diffuse reflection is then detected by a CMOS camera mounted in the solar field. Conversion of the measured gray values into a 2D flux density matrix is performed "online" by using the ratio of flux gauge measurement and surrounding gray values as calibration factor. An exemplary image is given in FIGURE 10. It shows both regions of interest (the aperture and the area on the passive insulated protection shield for evaluation) and the position of the flux gauge. Thus, the solar input power can be determined.

\section{TESTS}

\section{Commissioning}

Cold Commissioning has been carried out starting in July 2017 with I/O checks of all signals and tests of the particle system without particles. Therefore, all components like valves and drives have been checked and safety systems tested. In a second step, particles have been filled into the cold storage and the amount of particles fed into the system has been increased step by step until the desired performance has been achieved.

Cold particle receiver system tests have been conducted to ensure a safe and reliable operation. The water-cooling system for the front protection shield has been commissioned as well. Commissioning finished successfully in September 2017.

\section{Solar test}

Solar operation is started by accelerating the receiver to operational speed. In a second step, the particle mass flow is started to fill the receiver with particles. Then the number of heliostats is increased to heat up the system. The tests aim to primarily increase temperature at low mass flow and secondly increase power by increasing mass flow and solar input power.

Solar tests began on $24^{\text {th }}$ of September 2017 with a moderate operation of 27 heliostats being focused on the receiver. A receiver outlet temperature of $100^{\circ} \mathrm{C} / 212^{\circ} \mathrm{F}$ has been achieved during 3 hours of on-sun operation. In the following test days the number of focused heliostats has been increased step by step targeting higher loads and higher receiver outlet temperatures up to $900^{\circ} \mathrm{C} / 1,650^{\circ} \mathrm{F}$. Due to limitations in the heliostat field and low sun elevation angles in autumn, only tests with low solar input power could be realized up to now. Thus, high temperatures could only be achieved by operating at low particle mass flow of about $15-20 \%$ of the design mass flow of $0.7 \mathrm{~kg} / \mathrm{s}$. The rotational speed of the receiver has been maintained stationary. Both, mass flow and rotational speed, have been set to fixed values and not controlled automatically during the first test period.

FIGURE 6 shows the receiver during on-sun operation where the round aperture and the illuminated front protection shield can be observed.

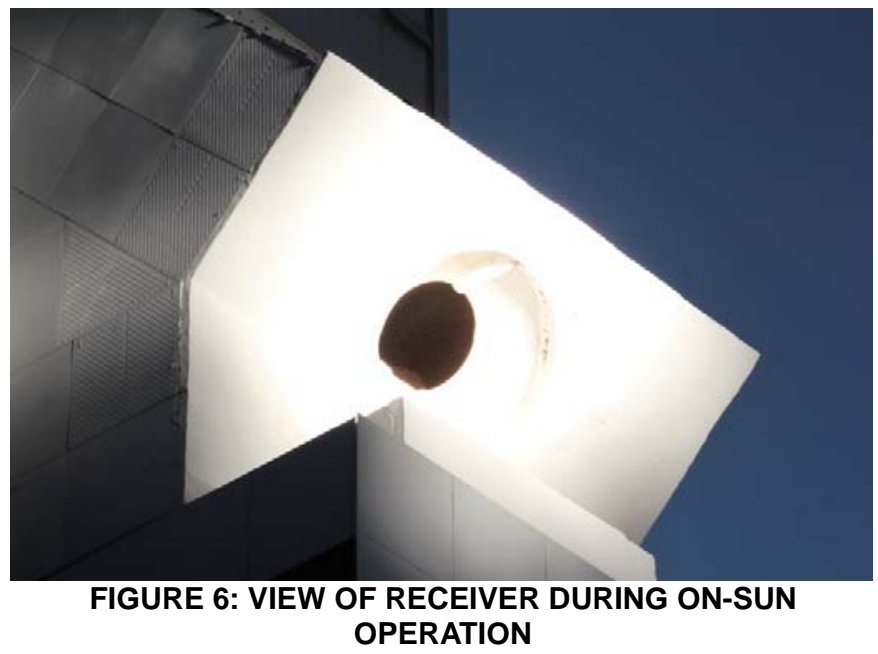

\section{RESULTS}

\section{Receiver}

TABLE 3 gives an overview of all conducted tests until October 2017. During five weeks 11 on-sun tests have been carried out achieving 28 hours of on-sun operation. The highest receiver outlet temperature $\mathrm{T}_{\mathrm{REC}, \mathrm{OUT}}$ of $775^{\circ} \mathrm{C} / 1,427^{\circ} \mathrm{F}$ has been reached on test day 9 on $18^{\text {th }}$ of October 2017.

FIGURE 7 gives an overview of the test on day 9 showing a sunny day with approximately $800 \mathrm{~W} / \mathrm{m}^{2}$ DNI and a maximum number $\mathrm{n}_{\text {Helio }}$ of more than 1,100 heliostats focused on the receiver. The rotational speed of the receiver and the particle mass flow have been maintained constant during the test with approximately $\omega_{\mathrm{REC}}=45 \mathrm{rpm}$ and an average mass flow $\dot{m}_{\text {particles }}$ of $0.15-0.18 \mathrm{~kg} / \mathrm{s}$, respectively. The flux density measured by a flux gauge (sensor position is given in 
FIGURE 10) shows flux peaks of up to $375 \mathrm{~kW} / \mathrm{m}^{2}$. Besides the receiver inlet and outlet temperature $T_{\text {REC,IN }}$ and $T_{\text {REC,OUT }}$ one exemplary sensor of the temperature $T_{\text {REC,SURFACE }}$ is also plotted. Although the maximum temperature $\mathrm{T}_{\mathrm{REC}, \mathrm{OUT}}$ measured in the outlet was $775^{\circ} \mathrm{C} / 1,430^{\circ} \mathrm{F}$, this value was obtained during an increasing trend so that under stationary conditions higher outlet temperatures are expected.

The test has been interrupted four times as the frequency converter of the receiver motor suffered an overload due to inadequate parameters. The problem with the frequency converter has been fixed thereafter by adjusting the parameters.

After every restart of the receiver a fast increase of the receiver outlet temperature can be observed with peak values of $50 \mathrm{~K} / \mathrm{min}$. The fourth attempt starting at approx. $14: 30 \mathrm{~h}$ is the longest heating period with the highest flux density measured. A significant temperature increase for several minutes of the $\mathrm{T}_{\text {REC,SURFACE }}$ sensor plotted in FIGURE 7 can be observed. This temperature increase cannot be observed at the receiver outlet

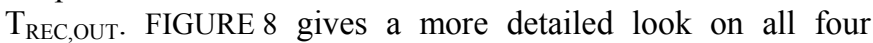
working sensors $\mathrm{T}_{\text {REC,SURFACE}}$, on the temperature $\mathrm{T}_{\text {REC,OUT, }}$ on the rotational speed $\omega_{\mathrm{REC}}$ and the particle mass flow $\dot{m}_{\text {particles }}$. The operation mode with the variation of the rotational speed with the corresponding particle film movement at lower speed and the resulting up and down in the receiver outlet temperature $\mathrm{T}_{\mathrm{REC} \text {,OUT }}$ can be observed. Three of the four temperatures $\mathrm{T}_{\text {REC,SURFACE }}$ showed the sudden temperature increase, but with a small time offset.

The reason for the temperature increase might be caused by local inhomogeneities in the particle film: After several hours of on-sun operation the point welded wire mesh has partly lifted off the surface and is not totally attached anymore like at the beginning (see FIGURE 9). This is probably caused by reduction of internal stresses in the woven wire mesh at high temperature. This may lead to small local inhomogeneities in the particle film and causes temperature changes. The mesh has been fixed after the on-sun test period in autumn. No further tests have been done after the repair so far.

TABLE 3 TEST OVERVIEW

\begin{tabular}{|r|c|c|c|}
\hline $\mathrm{N}^{\circ}$ & Date & $\begin{array}{c}\text { Operation } \\
\text { hours on-sun } \\
\text { HH:MM }\end{array}$ & $\begin{array}{c}\text { Max. receiver outlet } \\
\text { temperature } \mathrm{T}_{\text {REc;0ut }} \\
\text { in }{ }^{\circ} \mathrm{C} /{ }^{\circ} \mathrm{F}\end{array}$ \\
\hline 1 & 24.09 .2017 & $3: 01$ & $100 / 210$ \\
\hline 2 & 25.09 .2017 & $2: 52$ & $370 / 700$ \\
\hline 3 & 27.09 .2017 & $3: 20$ & $500 / 930$ \\
\hline 4 & 29.09 .2017 & $1: 07$ & $500 / 930$ \\
\hline 5 & 06.10 .2017 & $0: 32$ & $50 / 120$ \\
\hline 6 & 12.10 .2017 & $5: 10$ & $350 / 660$ \\
\hline 7 & 14.10 .2017 & $4: 00$ & $650 / 1,200$ \\
\hline 8 & 17.10 .2017 & $1: 42$ & $300 / 570$ \\
\hline 9 & 18.10 .2017 & $3: 16$ & $775 / 1,430$ \\
\hline 10 & 19.10 .2017 & $2: 17$ & $700 / 1,290$ \\
\hline 11 & 27.10 .2017 & $0: 51$ & $250 / 480$ \\
\hline & sum & $\mathbf{2 8 : 0 8}$ & \\
\hline
\end{tabular}

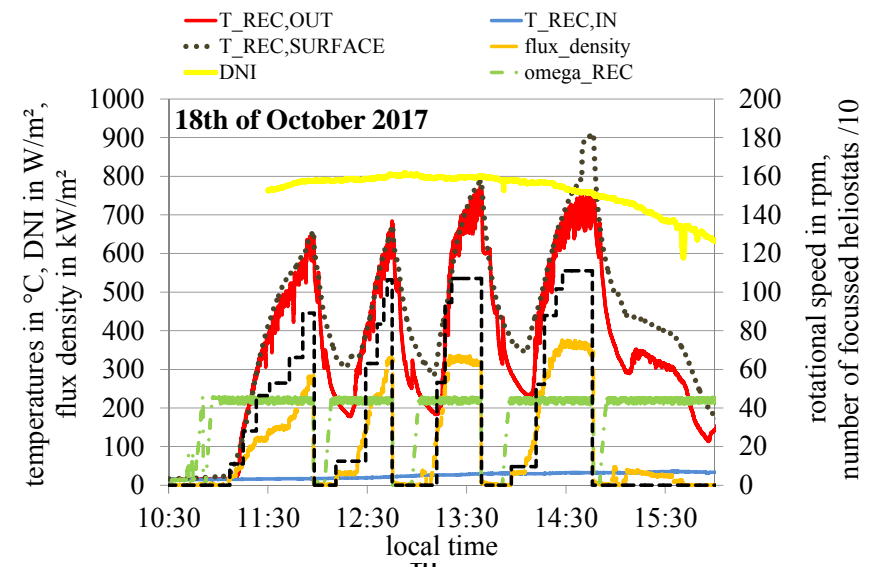

FIGURE 7: TEST DAY $18^{\text {TH }}$ OF OCTOBER 2017 WITH RECEIVER OUTLET TEMPERATURE OF $775^{\circ} \mathrm{C} / 1,430^{\circ} \mathrm{F}$

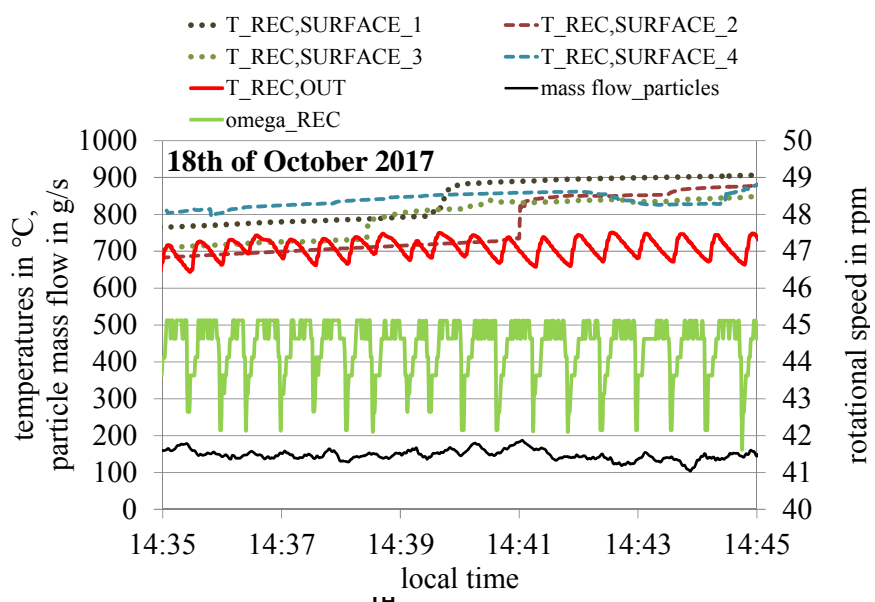

FIGURE 8: TEST DAY $18^{\text {TH }}$ OF OCTOBER 2017, DETAIL OF LAST 10 MINUTES BEFORE DEFOCUS

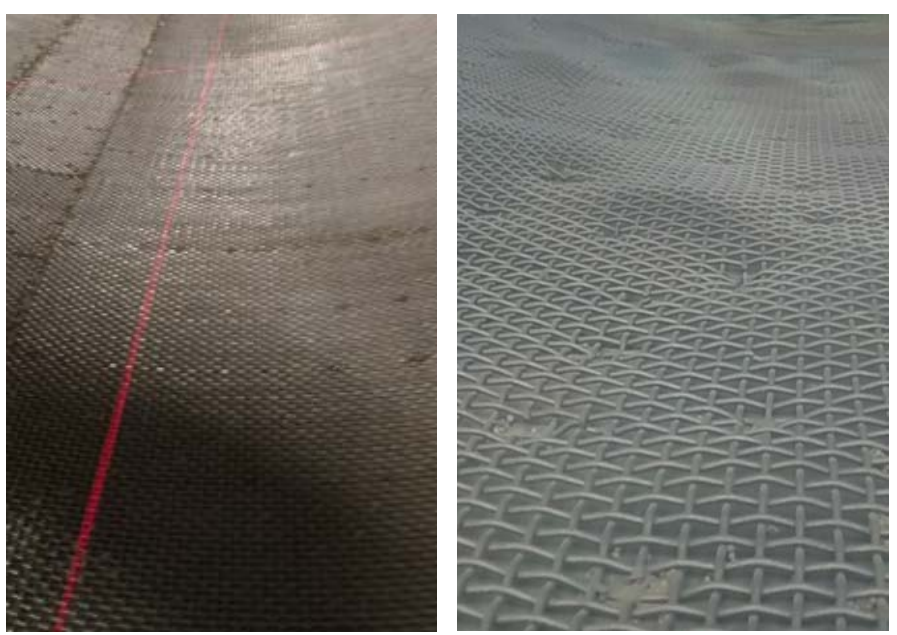

FIGURE 9: WIRE-MESH RECENTLY INSTALLED (LEFT) AND AFTER APROX. 30 H OF ON-SUN OPERATION (RIGHT) 
During the operation several flux density measurements like described above have been taken. An exemplary flux density image with a peak flux of approximately $300 \mathrm{~kW} / \mathrm{m}^{2}$ is given in FIGURE 10.

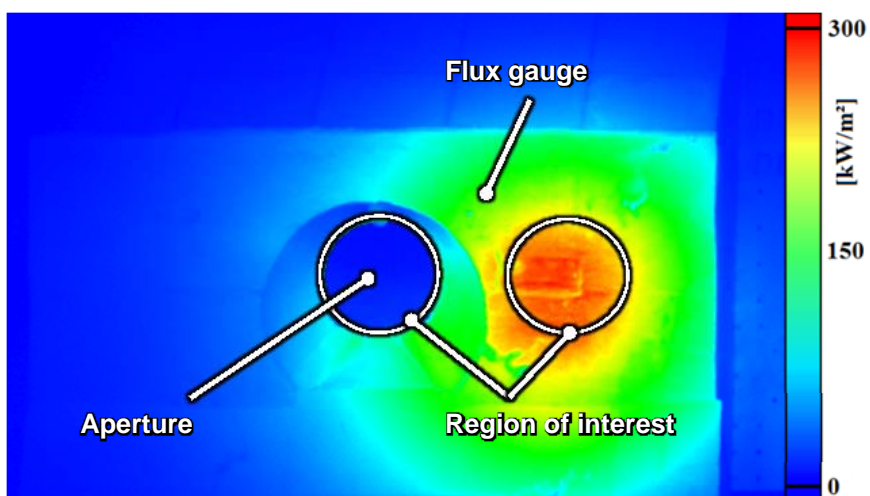

FIGURE 10: EXAMPLE FLUX DENSITY IMAGE TAKEN ON $17^{\mathrm{TH}}$ OF NOVEMBER 2017

Besides temperature measurements with thermocouples the particle film temperature distribution is measured with an infrared camera (ImageIR ${ }^{\circledR} 8380$ using a $3.5 \mu \mathrm{m}$ spectral bandpass filter) positioned in the heliostat field. An infrared image with the same view as the photo given in FIGURE 6 is presented in FIGURE 11. It shows the actively water-cooled and passive insulated front protection shield and part of the inner surface of the rotating receiver during operation. The emissivity $\varepsilon$ of the particles at high temperatures is not measured and the effect of reflections inside the cavity is not evaluated yet. Thus, $\varepsilon$ is determined by comparing the IR-image with the temperatures $T_{\text {REC,SURFACE }}$ measured inside the receiver and results to $\varepsilon=0.8$. Particle film temperatures of more than $900^{\circ} \mathrm{C} / 1,650^{\circ} \mathrm{F}$ can be observed at the lower end of the rotating part of the receiver close to the aperture. On two test days $\left(18^{\text {th }}\right.$ and $19^{\text {th }}$ of October 2017) temperatures $T_{\text {REC,SURFACE }}$ have surpassed $900^{\circ} \mathrm{C} / 1,650^{\circ} \mathrm{F}$. Although there might be small local inhomogeneities, in general a very homogenous distribution with approximately $50 \mathrm{~K}$ differences over the circumferences at a temperature level of $900^{\circ} \mathrm{C}-1,000^{\circ} \mathrm{C}\left(1,650^{\circ} \mathrm{F}-1,830^{\circ} \mathrm{F}\right)$ can be observed in the infrared image and measured with the thermocouples $T_{\text {REC,SURFACE }}$. Additionally, no spots with higher temperature are visible which would appear when part of the surface is not covered with particles.

The homogenous temperature distribution without spots indicates a homogenous particle film behavior and proves the functionality of the concept.

The temperature difference between receiver temperature $\mathrm{T}_{\text {REC,SURFACE }}$ and receiver outlet temperature $\mathrm{T}_{\mathrm{REC} \text {,OUT }}$ is still significant as tests have been carried out at very low mass flow and low thermal power. Thus, the stationary collector ring is still not totally heated up and heat losses in the collector ring are still relatively high compared to the overall low thermal power. This effect will decrease significantly while increasing thermal power, as temperature and losses in the collector ring will remain the same.

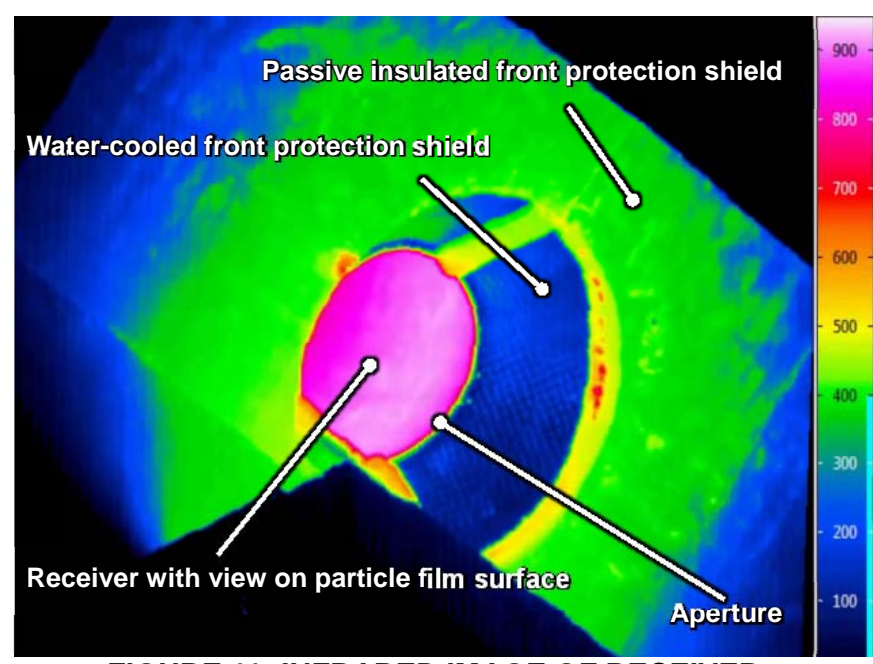

FIGURE 11: INFRARED IMAGE OF RECEIVER, TEMPERATURES IN ${ }^{\circ} \mathrm{C}\left(19^{\mathrm{TH}}\right.$ OF NOVEMBER, 1:18 PM WITH PEAK $T_{\text {REC,SURFACE }}>900^{\circ} \mathrm{C}$, EPSILON $=0.8$, VALID ONLY FOR PARTICLE SURFACE)

In general a very reliable operation has been observed and very high temperatures achieved in a short test period.

During the test period in autumn no stationary weather and test conditions could be achieved and no steady-state operation conducted. Therefore, no thermal efficiencies of the receiver have been evaluated up to now.

\section{System}

No major problem has been observed with the particle system. The bucket belt elevator has operated without problems during all tests. The automatic control system to refill the hopper based on the gravitational measurement has been working in a reliable way. The piping from the bucket belt elevator to the hopper showed a limitation in mass flow throughput. The limitation is probably caused by a widemeshed filter installed in front of the hopper. Dirt in the system clogs part of the filter and even small obstacles in the piping decrease the capacity of the piping significantly. This problem could be reduced by inserting an additional filter in the cold storage to maintain the particles as clean as possible and by installing a compressed air turbine vibrator on the piping. Also the pinch valve after the hopper worked well during the operation. The pinch valve is limited in temperature to a maximum of $80^{\circ} \mathrm{C} / 176^{\circ} \mathrm{F}$ due to the rubber sleeve. Thus, a small air cooling system has been required to ensure safe operation.

\section{CONCLUSION AND OUTLOOK}

A particle system has been successfully designed, manufactured, installed and commissioned in the Juelich Solar Tower. It allows the validation of the $\operatorname{CentRec} \AA$ receiver under real conditions in an overall test setup.

Up to now, a very promising startup of the CentRec $\mathbb{R}$ system has been reached in very short time. Temperatures of up to $775^{\circ} \mathrm{C} / 1,430^{\circ} \mathrm{F}$ have been achieved at the receiver outlet with 
even higher temperatures $>900^{\circ} \mathrm{C}$ inside of the receiver. A homogenous particle film and a robust operation have been observed allowing high solar fluxes and transients. The implemented point-welded wire mesh in the receiver is not adequate for long time operation at the given conditions and needs to be replaced in the further development by a perforated sheet or similar.

As the heliostat field at the Solar Tower Jülich is not optimized for small, high flux density receivers, tests can only be conducted up to $500 \mathrm{~kW} / \mathrm{m}^{2}$ flux density in the aperture and very high spillage can be observed during operation (see FIGURE 6). Additionally, sun elevation in October was already low. Due to these restrictions, high temperatures could only be achieved by reducing the mass flow significantly. No on-sun tests with higher mass flow and higher thermal power could be conducted up to now.

In spring 2018 further solar tests with different loads will be conducted. It is expected that a higher sun elevation angle allows achieving a thermal power of up to $500 \mathrm{~kW}$ and steadystate conditions so that the system performance can be evaluated. Receiver outlet temperatures of up to $900^{\circ} \mathrm{C} / 1,650^{\circ} \mathrm{F}$ are targeted. The test data and the evaluated performance will be used to validate a FEM model. With this model the thermal efficiency of a commercial design will be estimated. Tests will be conducted until summer 2018 .

\section{ACKNOWLEDGMENTS}

The development of the CentRec ${ }^{\circledR}$ technology was supported by the Helmholtz Validation Fund (grant no. HVF0028) as well as by DLR Technology Marketing and DLR's Energy Programme Directorate (grant no. LRV 16/113).

The authors would also like to thank Birgit Gobereit, David Trebing, Gereon Feckler, Andreas Krause, Tobias Prosinečki, Oliver Kaufhold, Björn Freitag, the operation team and all colleagues at the Juelich Solar Tower for their valuable contribution to the project.

\section{REFERENCES}

[1] L. Amsbeck, B. Behrendt, T. Prosin, R. Buck, "Particle tower system with direct absorption centrifugal receiver for high temperature process heat", Solarpaces 2014, Bejing, China

[2] Wei Wu, "A centrifugal Particle Receiver for HighTemperature Solar Applications", PhD RWTH Aachen 2014, Logos Verlag Berlin 2015, ISBN 978-3-8325-3882-8

[3] Ebert M., Amsbeck L., Jensch A., et al. Upscaling, Manufacturing and Test of a Centrifugal Particle Receiver. ASME. Energy Sustainability, V001T04A007. doi:10.1115/ES2016-59252.

[4] Lars Amsbeck, Reiner Buck, Miriam Ebert, Birgit Gobereit, Johannes Hertel, Andrea Jensch, Jens Rheinländer, David Trebing, Ralf Uhlig, "First Tests of a Centrifugal Particle Receiver with a $1 \mathrm{~m}^{2}$ Aperture", Solarpaces 2017, Santiago de Chile, Chile

[5] DLR, The Juelich solar tower research facility, URL: http://www.dlr.de/sf/en/desktopdefault.aspx/tabid-

8560/15527_read-44868/(16.11.2017)
[6] National Instruments Corporation: LabView. http://www.ni.com

[7] Sterz, Wladimir (2017) Entwicklung eines Strahlungsflussdichtemesssystems mit 'Moving Focus' für den Solarturm Jülich. Masterarbeit, RWTH Aachen.

[8] Röger, Marc und Herrmann, Patrik und Ebert, Miriam und Prahl, Christoph und Ulmer, Steffen und Göhring, Felix (2011) Flux Density Measurement on Large-Scale Receivers. In: Proc. of SolarPACES 2011. SolarPACES 2011, Sept. 20-23, 2011, Granada, Spain. 